\title{
Quiver of hyperstructures for Ying's twin universes
}

\author{
Thomas Vougiouklis \\ Democritus University of Thrace, School of Education, 68100 Alexandroupolis, Greece
}

\section{Email address:}

tvougiou@eled.duth.gr

\section{To cite this article:}

Thomas Vougiouklis. Quiver of Hyperstructures for Ying's Twin Universes. American Journal of Modern Physics. Special Issue: New Science Light Path on Cosmological Dark Matters. Vol. 4, No. 1-1, 2015, pp. 30-33. doi: 10.11648/j.ajmp.s.2015040101.16

Abstract: The quiver of hyperstructures, especially very large classes of them, can be used in new scientific theories such as Ying's twin universes. We present the largest class of hyperstructures which can be used as a model to represent the twin universe cosmos as even more new axioms or conditions are considered.

Keywords: Hyperstructure, $\mathrm{H}_{\mathrm{v}}$-Structure, Hope, Twin Universe

\section{Introduction}

Traveling in hyperstructures and their representations one can see some very beautiful landscape and fascinating site-views: $\mathrm{H}_{\mathrm{v}}$-groups, $\mathrm{H}_{\mathrm{v}}$-rings, $\mathrm{H}_{\mathrm{v}}$-fields, $\mathrm{H}_{\mathrm{v}}$-vector spaces, $\mathrm{H}_{\mathrm{v}}$-Lie algebras. We have to transfer as many possible results from classical theories, and since hyperstructure theory is very large, especially the weak ones, the problem becomes difficult but aesthetically beautiful. Weak axioms give larger classes and their associated applications. As new axioms appeared, new terms and constructions are introduced in order to manipulate and to classify the large classes of new hyperstructures.

The new theories in sciences, especially the revolutionary ones, need a very large class of structures in order that the researcher can select the most appropriate to express the theoretical background to improve the results. In this note I will present $\mathrm{H}_{\mathrm{v}}$-structures which can be used in the Ying's twin universe theories $[1,2,3]$ composed of two anti-symmetric systems which have two universes of 4-dimensional space-time coexisting on opposite sides of a 2-dimensional membrane.

\section{Basic Definitions}

Hyperstructures are called algebraic structures equipped with at least one hyperoperation (abbreviated to hope) [4]. Weak hyperstructures introduced in 1990 [5] are called $H_{v}$-structures and are defined as follows:

In a set $H$ equipped with a hope $H \times H \rightarrow \mathbf{P}(H)$, we abbreviate by WASS the weak associativity: $(x y) z \cap x(y z)=0, \forall x, y, z \in H$ and by $C O W$ the weak commutativity: $x y \cap y x \neq \emptyset, \forall x, y e H$.

The hyperstructure $(H, \cdot)$ is called $H_{v}$-semigroup if it is $W A S S$, is called $H_{v}$-group if it is reproductive $\mathrm{H}_{\mathrm{v}}$-semigroup: $x H=H x=H, \forall x \in H .(R,+, \cdot)$ is called $H_{v}$-ring if $(+)$ and $(\cdot)$ are WASS, the reproduction axiom is valid for $(+)$ and $(\cdot)$ is weak distributive with respect to $(+)$ :

$$
\begin{gathered}
x(y+z) \cap(x y+x z) \neq \emptyset,(x+y) z \cap(x z+y z) \neq \emptyset, \\
\forall x, y, z \in R
\end{gathered}
$$

For more definitions, results and applications on $\mathrm{H}_{\mathrm{v}}$-structures, see $[6,7,8,9,10]$. An extreme class is the following: an $\mathrm{H}_{\mathrm{v}}$-structure is very thin if all hopes are operations except one, with all hyperproducts singletons except only one, which is a subset with cardinality more than one.

The fundamental relations $\beta^{*}$ and $\gamma^{*}$ are defined, in $\mathrm{H}_{\mathrm{v}}$-groups and $\mathrm{H}_{\mathrm{v}}$-rings, respectively, as the smallest equivalences so that the quotient would be group and ring, respectively.

Theorem 2.1 Let $(H, \cdot)$ be $\mathrm{H}_{\mathrm{v}}$-group and let us denote by $U$ the set of all finite products of elements of $\mathrm{H}$. We define the relation $\beta$ in $H$ as follows: $x \beta y$ if $\{x, y\} \subset u$ where $\mathrm{u} \in U$. Then the fundamental relation $\beta^{*}$ is the transitive closure of the relation $\beta$.

An element is called single if its fundamental class is a singleton.

Motivation for $H_{v}$-structures:

(A)The quotient of a group with respect to an invariant subgroup is a group.

(B)F. Marty states that, the quotient of a group by any subgroup is a hypergroup. 
(C) Now, the quotient of a group with respect to any partition is an $\mathrm{H}_{\mathrm{v}}$-group.

Definition 2.2 Let $(H, \cdot),(H, \otimes)$ be $\mathrm{H}_{\mathrm{v}}$-semigroups defined on the same $H .(\cdot)$ is smaller than $(\otimes)$, and $(\otimes)$ greater than $(\cdot)$, if there exists automorphism $f \in A u t(H, \otimes)$ such that $x y \subset f(x \otimes y)$, $\forall x \in H$. Then $(H, \otimes)$ contains $(H, \cdot)$ and write $\leq \otimes \otimes$. If $(H, \cdot)$ is a structure, then it is basic and $(H, \otimes)$ is an $H_{b}$-structure.

The Little Theorem. Greater hopes of the ones which are $W A S S$ or $C O W$, are also WASS and $C O W$, respectively.

Definition $2.3[5,6]$ The $\mathrm{H}_{\mathrm{v}}$-ring $(R,+, \cdot)$ is an $H_{v}$-field if the quotient $R / \gamma *$ is a field.

Definitions $2.4[6]$ Let $(F,+, \cdot)$ be an $\mathrm{H}_{\mathrm{v}}$-field, $(V,+)$ be COW $\mathrm{H}_{\mathrm{v}}$-group and there exists an external hope $\because F \times V \rightarrow P(V)$ : $(\mathrm{a}, \mathrm{x}) \rightarrow \mathrm{ax}$ such that, $\forall \mathrm{a}, \mathrm{b} \in \mathrm{F}, \forall \mathrm{x}, \mathrm{y} \in \mathrm{V}$ we have

$\mathrm{a}(\mathrm{x}+\mathrm{y}) \cap(\mathrm{ax}+\mathrm{ay}) \neq \varnothing, \quad(\mathrm{a}+\mathrm{b}) \mathrm{x} \cap(\mathrm{ax}+\mathrm{bx}) \neq \varnothing, \quad(\mathrm{ab}) \mathrm{x} \cap \mathrm{a}(\mathrm{bx}) \neq \varnothing$,

then $V$ is called an $H_{v}$-vector space over $F$.

In the above cases the fundamental relation $\varepsilon^{*}$ is defined to be the smallest equivalence relation such that the quotient $\mathrm{V} / \varepsilon^{*}$ is a vector space over the field $\mathrm{F} / \gamma^{*}$.

The general definition of an $\mathrm{H}_{\mathrm{v}}$-Lie algebra was given in [11] as follows:

Definition 2.5 Let $(L,+)$ be $\mathrm{H}_{\mathrm{v}}$-vector space over the field $(\mathrm{F},+, \cdot), \varphi: \mathrm{F} \rightarrow \mathrm{F} / \gamma^{*}$, the canonical map and $\omega_{\mathrm{F}}=\{\mathrm{x} \in \mathrm{F}: \varphi(\mathrm{x})=$ 0 \}, where 0 is the zero of the fundamental field $\mathrm{F} / \gamma^{*}$. Similarly, let $\omega_{\mathrm{L}}$ be the core of the canonical map $\varphi^{\prime}: L \rightarrow L / \varepsilon^{*}$ and denote by the same symbol 0 the zero of $\mathrm{L} / \varepsilon^{*}$. Consider the bracket (commutator) hope:

$$
[,]: L \times L \rightarrow \mathrm{P}(\mathrm{L}):(\mathrm{x}, \mathrm{y}) \rightarrow[\mathrm{x}, \mathrm{y}]
$$

then $L$ is an $\mathrm{H}_{\mathrm{v}}$-Lie algebra over $\mathrm{F}$ if the following axioms are satisfied:

(L1) The bracket hope is bilinear, i.e.

$$
\begin{gathered}
{\left[\lambda_{1} \mathrm{x}_{1}+\lambda_{2} \mathrm{x}_{2}, \mathrm{y}\right] \cap\left(\lambda_{1}\left[\mathrm{x}_{1}, \mathrm{y}\right]+\lambda_{2}\left[\mathrm{x}_{2}, \mathrm{y}\right]\right) \neq \varnothing} \\
{\left[\mathrm{x}, \lambda_{1} \mathrm{y}_{1}+\lambda_{2} \mathrm{y}\right] \cap\left(\lambda_{1}\left[\mathrm{x}_{1} \mathrm{y}_{1}\right]+\lambda_{2}\left[\mathrm{x}, \mathrm{y}_{2}\right]\right) \neq \varnothing,} \\
\forall \mathrm{x}, \mathrm{x}_{1}, \mathrm{x}_{2}, \mathrm{y}, \mathrm{y}_{1}, \mathrm{y}_{2} \in \mathrm{L} \text { and } \lambda_{1}, \lambda_{2} \in \mathrm{F}
\end{gathered}
$$

(L2) $[\mathrm{x}, \mathrm{x}] \cap \omega_{\mathrm{L}} \neq \varnothing, \quad \forall \mathrm{x} \in L$

(L3) $([\mathrm{x},[\mathrm{y}, \mathrm{z}]]+[\mathrm{y},[\mathrm{z}, \mathrm{x}]]+[\mathrm{z},[\mathrm{x}, \mathrm{y}]]) \cap \omega_{\mathrm{L}} \neq \varnothing, \quad \forall \mathrm{x}, \mathrm{y} \in L$

\section{Classes of $\mathbf{H}_{\mathrm{v}}$-Structures}

Definition 3.1 [6]. Let (G,·) be groupoid, then for all P such that $\varnothing \neq \mathrm{P} \subset \mathrm{G}$, define the following hopes called $P$-hopes: $\forall \mathrm{x}, \mathrm{y} \in \mathrm{G}$

$$
\begin{gathered}
P: x P y=(x P) y \cup x(P y), P_{r}: x P_{r} y=(x y) P \cup x(y P), \\
P_{1}: x P_{1} y=(P x) y \cup P(x y) .
\end{gathered}
$$

The $(\mathrm{G}, \mathrm{P}),\left(\mathrm{G}, \mathrm{P}_{\mathrm{r}}\right),\left(\mathrm{G}, \mathrm{P}_{1}\right)$ are called P-hyperstructures. The most usual case is if $(\mathrm{G}, \cdot)$ is semigroup, then $\mathrm{xPy}=(\mathrm{xP}) \mathrm{y} \cup \mathrm{x}(\mathrm{Py})=\mathrm{xPy}$, and $(\mathrm{G}, \mathrm{P})$ is a semihypergroup.

Definitions 3.2 [8]. Let (G,) be groupoid (resp., hypergroupoid) and $\mathrm{f}: \mathrm{G} \rightarrow \mathrm{G}$ be a map. We define a hope ( $\partial$ ), called theta-hope or simply $\partial$-hope, on $\mathrm{G}$ as follows

$$
\mathrm{x} \partial \mathrm{y}=\{\mathrm{f}(\mathrm{x}) \cdot \mathrm{y}, \mathrm{x} \cdot \mathrm{f}(\mathrm{y})\}, \underset{ }{\forall \mathrm{x}, \mathrm{y} \in \mathrm{G} .(\text { resp. } \mathrm{x} \partial \mathrm{y}=(\mathrm{f}(\mathrm{x}) \cdot \mathrm{y}) \cup(\mathrm{x} \cdot \mathrm{f}(\mathrm{y}),}
$$

If $(\cdot)$ is commutative then $(\partial)$ is commutative. If $(\cdot)$ is $C O W$, then $(\partial)$ is $C O W$.

Let $(\mathrm{G}, \cdot)$ be groupoid (resp. hypergroupoid) and $\mathrm{f}: \mathrm{G} \rightarrow P(\mathrm{G})-\{\varnothing\}$ be multivalued map. We define $\partial$, on $\mathrm{G}$ as follows

$$
x \partial y=(f(x) \cdot y) \cup(x \cdot f(y), \forall x, y \in G
$$

Basic Property. If $(\mathrm{G}, \cdot)$ is a semigroup then, for every $\mathrm{f}$, the (ว) is WASS.

Let $(\mathrm{G}, \cdot)$ be a groupoid and $\mathrm{f}_{\mathrm{i}}: \mathrm{G} \rightarrow \mathrm{G}, \mathrm{i} \in \mathrm{I}$, be a set of maps on $\mathrm{G}$. We consider the map $\mathrm{f}_{\cup}: \mathrm{G} \rightarrow P(\mathrm{G})$ such that $\mathrm{f}_{\cup}(\mathrm{x})=\left\{\mathrm{f}_{\mathrm{i}}(\mathrm{x}) \mid \mathrm{i} \in \mathrm{I}\right\}$, called the union of the $\mathrm{f}_{\mathrm{i}}(\mathrm{x})$. We define the union 2 -hope, on $\mathrm{G}$ if we consider as map the $\mathrm{f}_{\cup}(\mathrm{x})$. A special case for a given map $f$, is to take the union of this with the identity map. We consider the map $\mathrm{f} \equiv \mathrm{f} \cup(\mathrm{id})$, so $\mathrm{f}(\mathrm{x})=\{\mathrm{x}, \mathrm{f}(\mathrm{x})\}, \forall \mathrm{x} \in \mathrm{G}, \quad$ which we call $b$ - $\partial$-hope. Then we have:

$$
\mathrm{x} \partial \mathrm{y}=\{\mathrm{xy}, \mathrm{f}(\mathrm{x}) \cdot \mathrm{y}, \mathrm{x} \cdot \mathrm{f}(\mathrm{y})\}, \forall \mathrm{x}, \mathrm{y} \in \mathrm{G} .
$$

In weak hyperstructures we can enlarge or reduce the underline set so that we can have $\mathrm{H}_{\mathrm{V}}$-structures with more properties [9]. Enlargements or reductions are more useful in representation theory if the obtain $\mathrm{H}_{\mathrm{v}}$-structures have the same fundamental structure.

Construction 3.3 The Attach Construction. Let $(\mathrm{H}, \cdot)$ be an $\mathrm{H}_{\mathrm{V}}$-semigroup and $\mathrm{v} \notin \mathrm{H}$. We extend the $(\cdot)$ into the set $\mathrm{H}=\mathrm{H} \cup\{\mathrm{v}\}$ as follows:

$$
\mathrm{x} \cdot \mathrm{v}=\mathrm{v} \cdot \mathrm{x}=\mathrm{v}, \forall \mathrm{x} \in \mathrm{H} \text {, and } \mathrm{v} \cdot \mathrm{v}=\mathrm{H} .
$$

The $(\mathrm{H}, \cdot)$ is an $h / v$-group, i.e. there is a reproductivity on classes, where $(\mathrm{H}, \cdot) / \beta^{*} \cong \mathrm{Z}_{2}$ and $\mathrm{v}$ is a single element.

The hyperstructure $(\mathrm{H}, \cdot)$ is called attach $h / v$-group of $(\mathrm{H}, \cdot)$.

Remarks. The core of $(\mathrm{H}, \cdot)$ is the set $\mathrm{H}$. All scalar elements of $(\mathrm{H}, \cdot)$ are also scalars in $(\mathrm{H}, \cdot)$ and any unit element of $(\mathrm{H}, \cdot)$ is also a unit of $(\mathrm{H}, \cdot)$. Finally, if $(\mathrm{H}, \cdot)$ is $C O W$ (resp. commutative) then $(\mathrm{H}, \cdot)$ is also $C O W$ (resp. commutative).

Definitions 3.4 Let $(\mathrm{H}, \cdot)$ be a hypergroupoid.

We say that we remove $\mathrm{h} \in \mathrm{H}$, if we simply consider the restriction of $(\cdot)$ on $\mathrm{H}-\{\mathrm{h}\}$.

We say that $\mathrm{h} \in \mathrm{H}$ absorbs $\mathrm{h} \in \mathrm{H}$ if we replace $\mathrm{h}$, whenever it appears, by $\mathrm{h}$.

We say that $h \in H$ merges with $h \in H$, if we take as product of $\mathrm{x} \in \mathrm{H}$ by $\mathrm{h}$, the union of the results of $\mathrm{x}$ with both $\mathrm{h}$ and $\mathrm{h}$, and consider $h$ and $h$ as one class, with representative $h$.

\section{4. $\mathrm{H}_{\mathrm{v}}$-Structures Defined on Small Sets}

To compare classes we can see on the problem of enumeration and classification of $\mathrm{H}_{\mathrm{v}}$-structures on the small sets. Recently we have interesting results by using computers. 
The problem is complicate in $\mathrm{H}_{\mathrm{v}}$-structures because we have great numbers. The partial order introduced in $\mathrm{H}_{\mathrm{v}}$-structures, restrict the problem in finding the minimal, up to isomorphisms, $\mathrm{H}_{\mathrm{v}}$-structures. In this direction we have results by Bayon \& Lygeros as the following [12]:

The number of $\mathrm{H}_{\mathrm{v}}$-groups with three elements is 1.026.462. The 7.926 are abelians and the rest are not; 1.013 .598 are cyclic and 12.864 are not, 16 are very thin.

Up to isomorphism, in sets with three elements there are: 6.494 minimal $\mathrm{H}_{\mathrm{v}}$-groups. 137 are abelians and the rest are not;

From the $\mathrm{H}_{\mathrm{v}}$-groups with 4 elements: the 8.028.299.905 are abelian $\mathrm{H}_{\mathrm{v}}$-groups, from which the 7.995.884.377 are cyclic.

In order to compare the numbers we see that: There are 10.614.362 classical abelian hypergroups, from which the 10.607 .666 are cyclic.

From the above it is clear that the number of the $\mathrm{H}_{\mathrm{v}}$-structures is enormous comparing to the classical hyperstructures.

\section{Applications}

A new application of the hyperstructure theory in social sciences combining the hyperstructure and fuzzy theory, is to replace in questionnaires the scale of Likert by the bar of Vougiouklis \& Vougiouklis [13]. The hyperstructures are used as an organized device. The suggestion is the following: In every question substitute the Likert scale with 'the bar' whose poles are defined with ' 0 ' on the left end, and ' 1 ' on the right end:

$$
0
$$

The subjects/participants are asked instead of deciding and checking a specific grade on the scale, to cut the bar at any point they feel expresses their answer to the specific question. The use of the bar of Vougiouklis \& Vougiouklis instead of a scale of Likert has several advantages during both the filling-in and the research processing. The length of the bar is according to the Golden Ratio $6.2 \mathrm{~cm}$. The hyperstructure theory, gives innovating new suggestions to connect finite groups of objects. These suggestions are obtained from properties and special elements inside the hyperstructure.

The Lie-Santilli isotopies born to solve Hadronic Mechanics problems. Santilli proposed [14, 15] a 'lifting'of the trivial unit matrix of a normal theory into a nowhere singular, symmetric, real-valued, new matrix. The original theory is reconstructed such as to admit the new matrix as left and right unit. The isofields needed correspond to $\mathrm{H}_{\mathrm{v}}$-structures called e-hyperfields which are used in physics or biology. Definition: Let $\left(\mathrm{H}_{\mathrm{o}},+, \cdot\right)$ be the attached $\mathrm{H}_{\mathrm{v}}$-field of the $\mathrm{H}_{\mathrm{v}}$-semigroup $(\mathrm{H}, \cdot)$. If $(\mathrm{H}, \cdot)$ has a left and right scalar unit e then $\left(\mathrm{H}_{0},+, \cdot\right)$ is e-hyperfield, the attached $H_{v}$-field of $(\mathrm{H}, \cdot)$.

In [14] a P-hope was introduces which is appropriate for e-hyperstructures:

Construction 5.1 Let (G,.) be abelian group and $\mathrm{P}$ any subset of $\mathrm{G}$ with more than one elements. We define the hyperoperation $\times_{P}$ as follows:

$$
\mathrm{xx}_{\mathrm{p}} \mathrm{y}= \begin{cases}\mathrm{x} \cdot \mathrm{P} \cdot \mathrm{y}=\{\mathrm{x} \cdot \mathrm{h} \cdot \mathrm{y} \| \mathrm{h} \in \mathrm{P}\} & \text { if } \mathrm{x} \neq \mathrm{e} \text { and } \mathrm{y} \neq \\ \mathrm{x} \cdot \mathrm{y} & \text { if } \mathrm{x}=\mathrm{e} \text { or } \mathrm{y}=\mathrm{e}\end{cases}
$$

we call this hope $P_{e}$-hope. The hyperstructure $\left(\mathrm{G}, \times_{\mathrm{P}}\right)$ is an abelian $\mathrm{H}_{\mathrm{v}}$-group.

\section{Ying's Twin Universe}

The new theories in sciences, especially the revolutionary ones, need a very large class of structures in order that the researcher could select the most appropriate to express the theoretical background and to improve the results. Therefore, from paragraph 4 , it is clear that one can select the appropriate model from the huge number of the offered $\mathrm{H}_{\mathrm{v}}$-structures.

The Ying's Twin Universe [1, 2, 3] is the Total Universe which is composed of two anti-symmetric systems which have two universes of 4-dimensional space-time coexisting on opposite sides of a 2-dimensional membrane. That means than it has a Positive Universe and a Negative Universe and together with the membrane gives a total of 10-dimensions 'Cosmos'. Consequently, in the model we suggest to use:

1. An appropriate hyperfield from the class of $\mathrm{H}_{\mathrm{v}}$-fields to take the hypernumbers.

2. Two weak homomorphic 4-dimensional hyper- $\left(\mathrm{H}_{\mathrm{v}^{-}}\right)$-space-time coexisting on opposite sides over the above $\mathrm{H}_{\mathrm{v}}$-fields.

3. A 2-dimensional hyper- $\left(\mathrm{H}_{\mathrm{v}^{-}}\right)$-space.

4. Weak homomorphisms to transfer properties from one $\mathrm{H}_{\mathrm{v}}$-space-time to the other especially on the fundamental classes.

All the above generalizations must have as a special case the single value representation on the corresponding fundamental structures. Adding new axioms and conditions will restrict the cases of $\mathrm{H}_{\mathrm{v}}$-fields.

We suppose that we must have two weak homomorphic 4-dimensional hyper $\left(\mathrm{H}_{\mathrm{v}}\right)$ spacetime coexisting in order the model to admit some different cases on the opposite sides. This means that we can have even different axioms provided only that on the fundamental classes we have an ordinary Universe. In other words, we can partition on the Universes so that to have homomorphic models. To simplify the model we can use only one $\mathrm{H}_{\mathrm{v}}$-field, with fundamental field the complex field and to take the Cartesian product or the Cayleyen construction.

\section{References}

[1] L. Ying, Nuclear fusion drives cosmic expansion, Springer Proceedings in Physics: Springer, vol. 137, 2011, pp. 379-381.

[2] L. Ying, Anti-antimatter, Journal of Computational Methods in Sciences and Engineering, vol. 13, 2013, pp. 303-305.

[3] L. Ying, Twin universes: universal laws of thermodynamics, American Journal of Modern Physics, vol. 4, no. 3, 2015, pp. $1-4$. 
[4] F. Marty, Sur une generalization de la notion de groupe, $8^{\text {th }}$ Congress of Mathematicienes Scandinaves, Stockholm, 1934, pp. 45-49.

[5] T. Vougioukli, The fundamental relation in hyperrings. The general hyperfield, $4^{\text {th }}$ AHA, Xanthi 1990 , World Scientific, 1991, pp. 203-211.

[6] T. Vougiouklis, Hyperstructures and their representations, Hadronic Press, Florida, 1994.

[7] T. Vougiouklis, Some remarks on hyperstructures, Contemporary Mathematics, American Mathematical Society, vol. 184, 1995, pp. 427-431.

[8] T. Vougiouklis, $\partial$-operations and $\mathrm{H}_{\mathrm{v}}$-fields, Acta Math. Sinica, vol. 24, no. 7, 2008, pp. 1067-1078.

[9] T. Vougiouklis, On $\mathrm{H}_{\mathrm{v}}$-rings and $\mathrm{H}_{\mathrm{v}}$-representations, Discrete Mathematics, Elsevier, 208/209, 1999, pp. 615-620.

[10] T. Vougiouklis and S. Vougiouklis, The helix hyperoperations, Italian Journal of Pure and Applied Mathematics, vol. 18, 2005, pp. 197-206.
[11] T. Vougiouklis, The Lie-Hyperalgebras and their fundamental relations, Southeast Asian Bull. Maths., vol. 37., no. 4, 2013, pp. 601-614.

[12] R. Bayon and N. Lygeros, Advanced results in enumeration of hyperstructures, J. Algebra, vol. 320, 2008, pp. 821-835.

[13] T. Vougiouklis and P. Kambaki-Vougiouklis, On the use of the bar, China-USA Business Review, vol. 10, no. 6, 2011, pp. 484-489.

[14] D. Davvaz, R.M. Santilli, T. Vougiouklis, Multi-valued hypermathematics for characterization of matter and antimatter systems, J. Computational Methods in Sciences and Engineering vol. 13, 2013, pp. 37-50.

[15] R.M. Santilli and T. Vougiouklis, Lie-admissible hyperalgebras, Italian J. Pure Applied Math., vol.31, 2013, pp. 239-254. 